\title{
Effect of silk fibroin nanofibers containing silver sulfadiazine on wound healing
}

\author{
This article was published in the following Dove Press journal: \\ International Journal of Nanomedicine \\ 14 November 2014 \\ Number of times this article has been viewed
}

\section{Lim Jeong' \\ Min Hee Kim' \\ Ju-Young Jung ${ }^{2}$ \\ Byung Moo $\mathrm{Min}^{3}$ \\ Won Ho Park'}

'Department of Advanced Organic Materials and Textile System Engineering, ${ }^{2}$ College of Veterinary Medicine, Chungnam National University, Daejeon, ${ }^{3}$ Department of Oral Biochemistry, School of Dentistry, Seoul National University, Seoul, South Korea
Correspondence: Won Ho Park Department of Advanced Organic Materials and Textile System Engineering, Chungnam National University, Daejeon 305-764, South Korea Tel +82428216613

Fax +8242823 3736

Email parkwh@cnu.ac.kr
Background: One of the promising applications of silk fibroin (SF) in biomedical engineering is its use as a scaffolding material for skin regeneration. The purpose of this study was to determine the wound healing effect of SF nanofibrous matrices containing silver sulfadiazine (SSD) wound dressings.

Methods: An SF nanofibrous matrix containing SSD was prepared by electrospinning. The cell attachment and spreading of normal human epidermal keratinocytes (NHEK) and normal human epidermal fibroblasts (NHEF) to SF nanofibers containing three different concentrations of SSD contents $(0.1,0.5$, and $1.0 \mathrm{wt} \%)$ were determined. In addition, a rat wound model was used in this study to determine the wound healing effect of SF nanofibers containing SSD compared with that of Acticoat ${ }^{\mathrm{TM}}$, a commercially available wound dressing.

Results: The number of NHEK and NHEF attached to SF nanofibers containing SSD decreased when the concentration of SSD increased. The number of attached NHEF cells was lower than that of attached NHEK cells. The SF matrix with $1.0 \mathrm{wt} \% \mathrm{SSD}$ produced faster wound healing than Acticoat, although $1.0 \mathrm{wt} \%$ SSD inhibited the attachment of epidermal cells to SF nanofibers in vitro.

Conclusion: The cytotoxic effects of SF nanofibers with SSD should be considered in the development of silver-release dressings for wound healing through its antimicrobial activity. It is challenging to design wound dressings that maximize antimicrobial activity and minimize cellular toxicity.

Keywords: silk fibroin, silver sulfadiazine, nanofibrous matrix, wound healing

\section{Introduction}

The use of silver (Ag) as an antimicrobial agent has a long history. ${ }^{1}$ Since Moyer et al's use of $0.5 \%$ silver nitrate $\left(\mathrm{AgNO}_{3}\right)$ solution for patients with burns, use of $\mathrm{Ag}$ has resurged recently. ${ }^{2}$ Moyer proposed that $\mathrm{AgNO}_{3}$ solution did not interfere with epidermal proliferation, but possessed antibacterial properties against Staphylococcus aureus, Pseudomonas aeruginosa, and Escherichia coli. ${ }^{3,4}$ In 1968, $\mathrm{AgNO}_{3}$ was combined with sulfonamide to form silver sulfadiazine (SSD) cream, which served as an antibacterial agent with a broad spectrum of action. SSD cream, especially in the format of $1 \%$ cream, has been used for the treatment of burns. ${ }^{5}$

Among the various combinations of sulfa drugs with Ag tested in vitro, SSD appeared to be the most effective. ${ }^{6} \mathrm{SSD}$ is effective against various bacteria, such as E. coli, S. aureus, Klebsiella species, and Pseudomonas species. SSD is also reported to possess some antifungal and antiviral activity. ${ }^{7}$

Ag can be effective against a wide range of micro-organisms, including aerobic and anaerobic bacteria, fungi, and viruses. The antimicrobial effect of Ag involves several mechanisms: it interferes with the respiratory chain in the cytochromes of 
microbacteria $;{ }^{8}$ it interferes with components of the microbial electron transport system; ${ }^{9}$ and it binds DNA and inhibits DNA replication. ${ }^{10}$

Recently, there has been a rapid increase in the number of commercially available Ag dressings, such as $\mathrm{AgNO}_{3}$, SSD, and nanocrystalline Ag. Traditionally, the active Ag agent has been thought to be ionic Ag. However, elemental Ag was found to be active even when in a nanocrystalline form. ${ }^{11}$ It is possible to produce pure Ag particles at a nanometer scale with advanced nanotechnology. When cells or tissues are exposed to Ag nanoparticles (NPs), their active surface is significantly larger than that of other $\mathrm{Ag}$ compounds. As a result, Ag NPs are able to exhibit unusual physicochemical properties with remarkable biological activity. Ideal antimicrobial wound dressings should have more controlled and prolonged release of Ag compared with cream formulations during their entire period of usage. This will result in less frequent dressing changes, thereby reducing the risk of nosocomial infection, cost of care, further tissue damage, and patient discomfort. Many factors affect the clinical performance of a dressing, such as the Ag content, the chemical and physical forms of Ag, the distribution of $\mathrm{Ag}$, and its affinity for moisture..$^{8,12}$ The number of commercially available Ag-based dressings has increased recently in the medical market.

Silk fibroin (SF) has been widely used for cosmetics and food additives. Recently, SF has been found to have potential in the area of biomedical science and engineering due to its distinctive biological properties, including biocompatibility, oxygen and water vapor permeability, biodegradability, and minimally induced inflammatory responses in vivo. ${ }^{13-16}$ One of the promising applications of SF in biomedical engineering is its use as a scaffolding material for skin regeneration. It was reported that SF could be useful for the culture of fibroblasts and keratinocytes, because it could enhance adhesion, growth, and differentiation of cells with benefits similar to those afforded by collagen matrices. ${ }^{17-19}$ In addition, nanofibrous scaffolds of biocompatible SF have great potential as dressings for burns wounds when combined with Ag compounds (eg, SSD) because they have a high specific surface area and microporous structure, and show good adhesion to damaged skin.

In this study, SF nanofibers containing SSD were prepared as antimicrobial wound dressings. Re-epithelialization and wound contraction, the two important components in the process of wound healing, are mediated by keratinocytes and fibroblasts, respectively. Therefore, the cell attachment and spreading of normal human epidermal keratinocytes
(NHEK) and normal human epidermal fibroblasts (NHEF) to the SF nanofibers containing SSD were determined in this study. The effect of SF nanofibers containing SSD on wound healing was compared with that of Acticoat ${ }^{\mathrm{TM}}$ in an animal wound model.

\section{Materials and methods Materials}

Raw silk was obtained from the cocoons of the silkworm Bombyx mori. SSD $\left(\mathrm{C}_{10} \mathrm{H}_{9} \mathrm{AgN}_{4} \mathrm{O}_{2} \mathrm{~S} ; 99 \%\right)$ and formic acid (98\%) were purchased from Sigma-Aldrich (St Louis, MO, USA) and Junsei (Tokyo, Japan), respectively. Calcium chloride and ethanol were purchased from Samcheon Chemicals (Gyeonggi, Korea). Acticoat Ag-based dressings were purchased from Smith \& Nephew (London, UK).

\section{Preparation of electrospun SF nanofibers containing SSD}

The raw silk fibers were degummed with $0.5 \mathrm{wt} \%$ sodium bicarbonate $\left(\mathrm{NaHCO}_{3}\right)$ solution at $100^{\circ} \mathrm{C}$ for 30 minutes, followed by rinsing with warm distilled water. The degummed silk (SF) was dissolved in a ternary solvent system of calcium chloride/ethanol/water $\left(1 / 2 / 8\right.$ in molar ratio) at $70^{\circ} \mathrm{C}$ for 4 hours. After dialysis in distilled water for 3 days in a cellulose tubular membrane (molecular cutoff, 12,000-14,000), the aqueous SF solution was filtered and freeze-dried to obtain the regenerated SF sponge.

SF solution ( $8 \mathrm{wt} \%$ ) was prepared by dissolving the regenerated SF sponge in 98\% formic acid for 2 hours. $\mathrm{Ag}$ compounds were then added and dissolved in SF/formic acid solution for 1 hour. The concentrations of SSD were in the range of $0.1-1.0 \mathrm{wt} \%$ of the SF weight. The SF solution containing SSD was delivered using a syringe pump (KD Scientific, Holliston, MA, USA) at a flow rate of $0.5 \mathrm{~mL} /$ hour. The distance between the needle tip and the ground electrode was $8 \mathrm{~cm}$. The positive voltage applied to the polymer solutions was $16 \mathrm{kV}$. The electrospun SF nanofibers containing SSD were treated with water vapor in order to achieve solvent-induced crystallization of fibroin. The solvent-vapor treated samples were prepared by placing SF nanofiber matrices in a desiccator saturated with water vapor for 4 hours, followed by vacuum drying at room temperature for 24 hours.

\section{Cells and cell culture}

NHEK were prepared and maintained using a previously published procedure. ${ }^{18}$ Briefly, NHEK were isolated from human foreskin obtained surgically. The epidermal 
keratinocytes were isolated from separated epithelial tissue after trypsinization. The primary cell cultures were established in a keratinocyte growth medium containing $0.15 \mathrm{mM}$ calcium and a supplementary growth-factor bullet kit. Primary NHEK were plated at $1 \times 10^{5}$ cells per $60 \mathrm{~mm}$ culture dish and cultured until the cells reached $70 \%$ confluence. The second-passage keratinocyte cells were used in this study.

Primary NHEF were established from the explant cultures of foreskin connective tissue, which was excised from a patient undergoing surgery. The cells that proliferated outwardly from the explant culture were cultured continuously in Dulbecco's modified Eagle's medium supplemented with $10 \%$ fetal bovine serum. The third-passage fibroblast cells were used in this study.

All procedures used for sampling human tissue or specimens were performed in accordance with the guidelines of the institutional review board on human subject research, and the ethics committee at Seoul National University Dental Hospital, Seoul, Korea.

\section{Cell attachment and spreading assays}

The cell attachment assay was performed using the method reported by Mould et al with slight modifications. ${ }^{20}$ Briefly, SF nanofibers containing SSD were cut out with a punch (14 mm inner diameter) and placed onto 24-well cell culture plates. These plates were soaked in serum-free medium for 30 minutes at room temperature, followed by rinsing with phosphate-buffered saline (PBS). The cells were detached from the culture plates by treatment with $0.05 \%$ trypsin and $0.53 \mathrm{mM}$ ethylenediamine tetraacetic acid in PBS, and then resuspended in the culture medium. The cells were placed on the SF nanofibers containing SSD in each well at a density of $0.6 \times 10^{5}$ cells $/ 250 \mu \mathrm{L}$ and incubated at $37^{\circ} \mathrm{C}$ for 1 hour. The unattached cells were removed by rinsing twice with PBS. The attached cells were fixed with $10 \%$ formalin in PBS for 15 minutes and rinsed twice with PBS. The cells attached to the SF nanofibers containing SSD were stained with hematoxylin and eosin, and the wells were rinsed gently three times with double-distilled water. The SF nanofibers containing SSD were mounted and the cells attached to the matrix were photographed using an Olympus BX51 microscope.

The extent of cell spreading was analyzed using photographs obtained from the cell attachment assay. To ensure a representative count, each sample was divided into quarters, and two fields per quarter were photographed. The cells with a flattened, polygonal shape with filopodia-like and lamellipodia-like extensions were considered as spreading cells. In contrast, the cells that resisted washing and remained tethered to the plate surface were classified as nonspreading cells. Each spreading cell was determined using a computer equipped with CellProfiler cell image analysis software (Broad Institute, Cambridge, MA, USA). A minimum of 100 cells were counted on each occasion. The mean percentage and standard deviation were calculated from data obtained from four independent experiments. The percentage of cells showing a spreading morphology was quantified by dividing the number of spreading cells by the total number of bound cells.

\section{Animals}

Specific pathogen-free inbred male Sprague-Dawley (SD) rats, aged 7 weeks and routinely screened serologically for relevant respiratory pathogens, were purchased from Daehan Biolink Co Ltd (Seoul, Korea). Thirty-six SD rats were randomly divided into six groups, which were subjected to treatment with SF nanofibers only (no SSD; SF only), SF nanofibers containing $0.1 \mathrm{wt} \% \mathrm{SSD}$ (SF/AgS 0.1), SF nanofibers containing $0.5 \mathrm{wt} \% \mathrm{SSD}$ (SF/AgS 0.5), SF nanofibers containing $1.0 \mathrm{wt} \% \mathrm{SSD}(\mathrm{SF} / \mathrm{AgS} 1.0)$, Acticoat, or no treatment (control). The rats were maintained and acclimated for 1 week in an animal facility under standard laboratory conditions for this study, with water and standard provided ad libitum. All experimental procedures were performed in accordance with the National Institutes of Health Guidelines for the Care and Use of Laboratory Animals, and the animals were handled according to the dictates of the National Animal Welfare Laws of Korea.

\section{Surgical procedures for wound healing test}

The animals were anesthetized with an intramuscular injection of a mixture of Zoletil ${ }^{\circledR} 50$ (Virbac, Carros, France) and Rompun $^{\circledR}$ (Bayer Korea, Seoul, Korea; 150 cc/per rat). After anesthesia, the dorsal hair was carefully shaved. Four sites of full-thickness skin wounds above the fascia on the dorsal side of the rats were surgically created by a $6 \mathrm{~mm}$ diameter biopsy punch (Kai Industries, Seki, Japan). The wounds were treated with test dressings of SF only, SF/AgS 0.1, $\mathrm{SF} / \mathrm{AgS} 0.5, \mathrm{SF} / \mathrm{AgS} 1.0$, or Acticoat, and then covered with Tegaderm ${ }^{\mathrm{TM}}$ (3M, St Louis, MO, USA) to hold the dressings in place. The wounds in the control group were covered with Tegaderm only. The wound dressings and covers were replaced at days 3,7 , and 14 post wounding until sacrifice or wound healing. 


\section{Assessment of wound closure}

Wound closure was measured by a ruler and documented by digital photography before the animals were sacrificed at days 3,7 , and 14 post wounding. The wound healing rate was calculated as a percentage of the size of the unhealed wound area compared with the size of the initial dorsal wound area.

\section{Histologic and histomorphometric procedures}

At days 3, 7, and 14 post wounding, animals from each group were sacrificed with an overdose of a mixture of Zoletil 50 and Rompun. The original wound skins were harvested and fixed in $4 \%$ formalin overnight at $4{ }^{\circ} \mathrm{C}$. After fixation, the skins were embedded in paraffin and transversely cut into $5 \mathrm{~mm}$ thick slices, which were prepared with a microtome. Staining was performed using hematoxylin and eosin. Sections were analyzed using an optical microscope (Nikon, Tokyo, Japan) at 40× magnification. The extent of re-epithelialization of wounds was measured as the length of the line from the base of the dermis to the outlining of the epidermis from each section using the image analysis program (NIS-Elements, Nikon).

\section{Results and discussion Morphology of SF nanofibers containing SSD}

The SF nanofibers containing SSD were fabricated by electrospinning, and were composed of a randomly arranged fibrous structure, as shown in Figure 1A. The average fiber diameter of the SF only nanofibers was $379 \mathrm{~nm}$, which was determined by image analysis of the scanning electron microscopic images (not shown). The addition of SSD to the SF solution caused a significant increase in the average fiber diameter to $512 \mathrm{~nm}$ (Figure 1B). Energy dispersive spectroscopy analysis of the scanning electron microscopic images of SF nanofibers containing SSD is shown in Figure 1C. The elemental Ag and S in SSD were detected in the energy dispersive spectra.

\section{Cytotoxic effect of SF nanofibers containing SSD on cell adhesion and spreading}

In general, wounds produced in the dermis are healed by mechanisms involving re-epithelialization, construction, and matrix deposition. NHEK and NHEF both play important roles in the process of wound healing, because keratinocytes

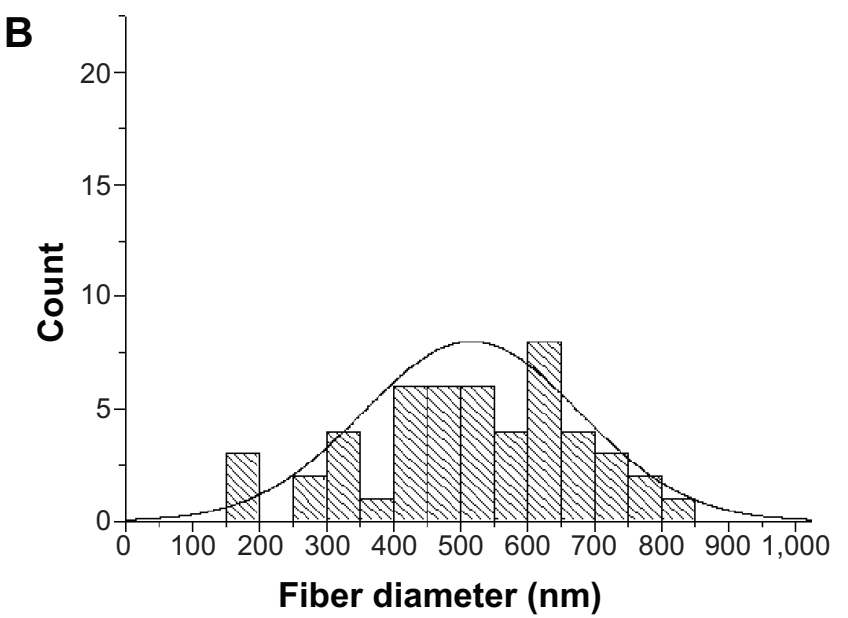

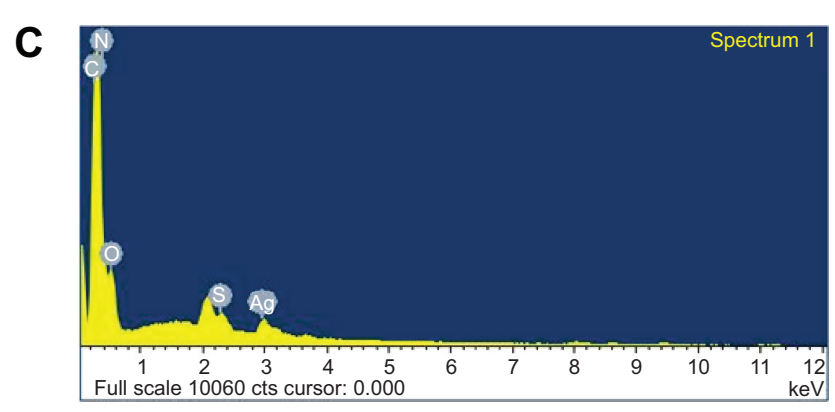

Figure I Scanning electron microscopic images (A), fiber diameter distribution (B), and energy dispersive spectra (C) of silk fibroin nanofibers containing silver sulfadiazine (1.0 wt\%). 
and fibroblasts are the main cell types involved in production of the dermal matrix. ${ }^{21}$ The initial cell attachment and spreading of the SF nanofibers containing Ag compounds were examined in this study because initial cell attachment and spreading may be important factors in the early stages of wound healing. To assess cell attachment, SF nanofibers containing SSD were seeded with NHEK and NHEF. The effect of SF nanofibers containing SSD on the attachment and spreading of NHEK is summarized in Figure 2. Representative images of NHEK attached to the SF nanofibers containing SSD are shown in Figure 2A. A significantly $(P<0.05)$ high number of NHEK adhered to the SF only nanofibers than to SF in the presence of SSD (Figure 2B). The number of NHEK attached to SF nanofibers containing $1.0 \mathrm{wt} \% \mathrm{SSD}$ was significantly lower than that to SF nanofibers containing 0.1 or $0.5 \mathrm{wt} \% \mathrm{SSD}$. When $1.0 \mathrm{wt} \%$
A

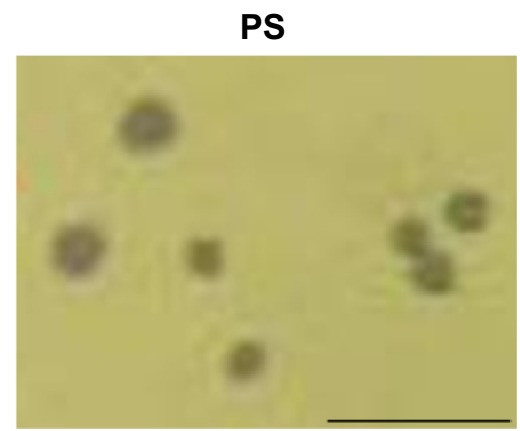

SF only

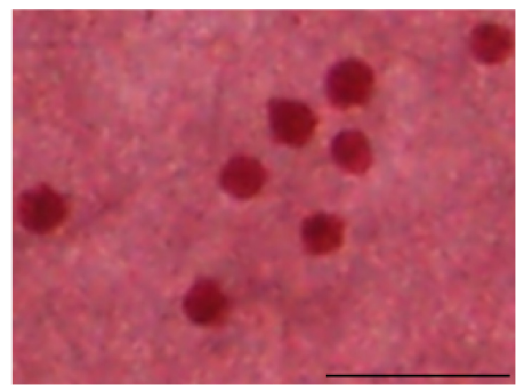

SFIAgS 0.5
SFIAgS 0.1

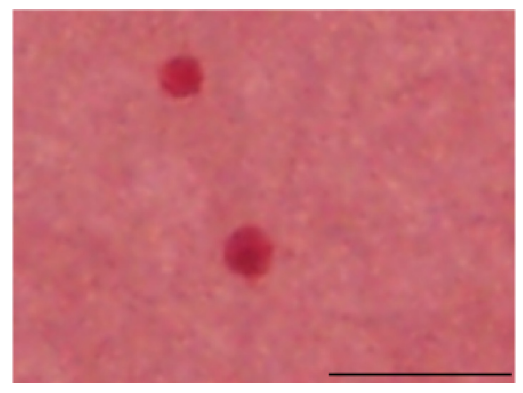

SFIAgS 1.0

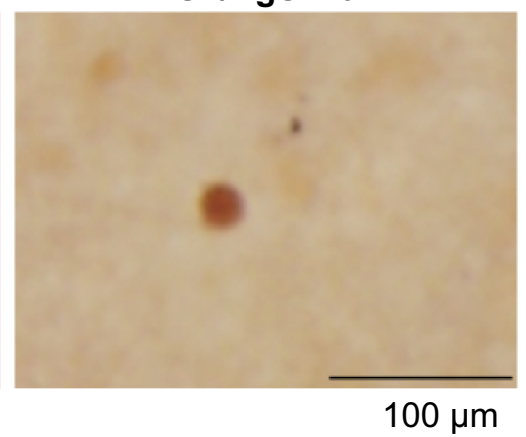

B

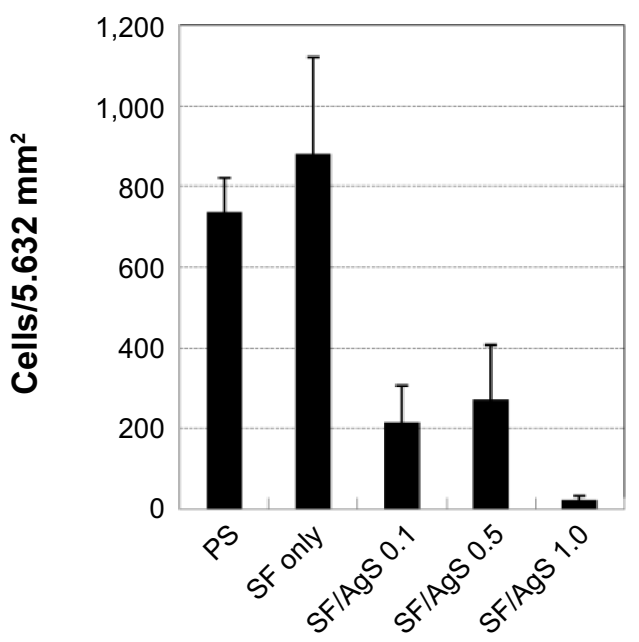

C

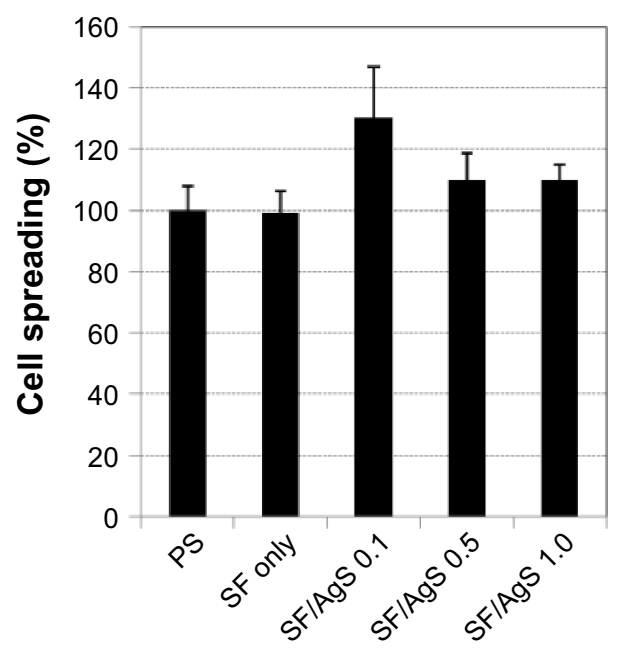

Figure 2 Effect of SF nanofibers containing SSD on the attachment and spreading of NHEK.

Notes: (A) Representative images of NHEK attached to SF nanofibers containing SSD. (B) Numbers of NHEK adhering to SF nanofibers containing SSD. (C) Percentage of cell spreading of NHEK plated onto SF nanofibers containing SSD. Data are presented as the mean \pm standard deviation $(n=4)$.

Abbreviations: Ag, silver; NHEK, normal human epidermal keratinocytes; PS, polystyrene tissue culture plate; SSD, silver sulfadiazine; SF, silk fibroin; AgS 0. I, 0.I wt\% SSD; AgS 0.5, 0.5 wt\% SSD; AgS I.0, I.0 wt\% SSD. 
SSD was added, it was too difficult for NHEK to attach to the SF nanofibers. The initial spreading of NHEK adhering to the SF nanofibers was similar in all groups, regardless of the SSD concentration (Figure 2C). Similarly, the number of NHEF attached to the SF nanofibers containing SSD was significantly $(P<0.05)$ lower than that attached to SF nanofibers only (Figure 3). The initial spreading of NHEF adhering to the SF nanofibers containing SSD gradually decreased when SSD concentrations were increased (Figure 3C). Taken together, these results indicate that SF nanofibers containing SSD were cytotoxic to both NHEK and NHEF.

\section{Skin wound healing effect of SF nanofibers containing SSD}

Wound healing is a complex and multistep process involving the integration of a variety of tissue and cell types. ${ }^{22}$ Re-epithelialization, a crucial process during the early phase of wound healing, occurs not only by the migration
A

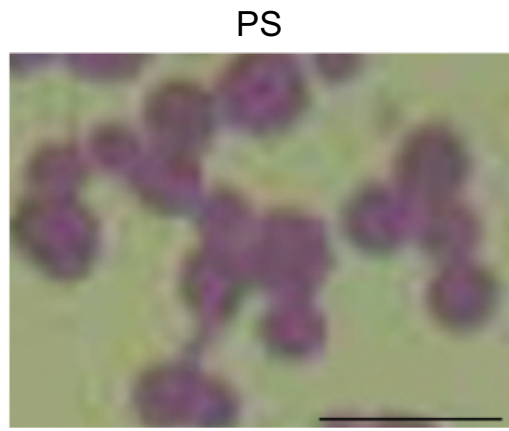

SF only

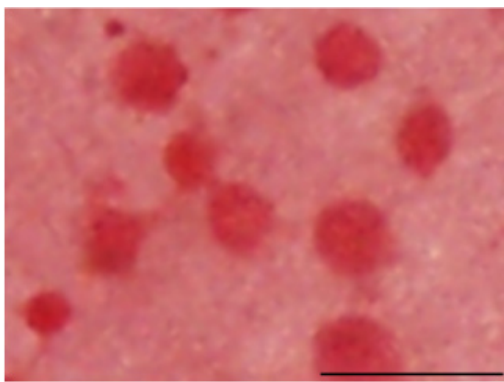

SF/AgS 0.5

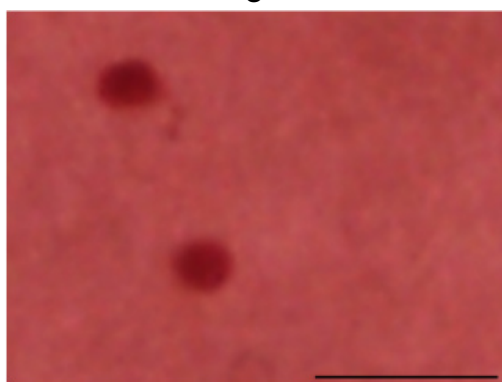

SF/AgS 0.1

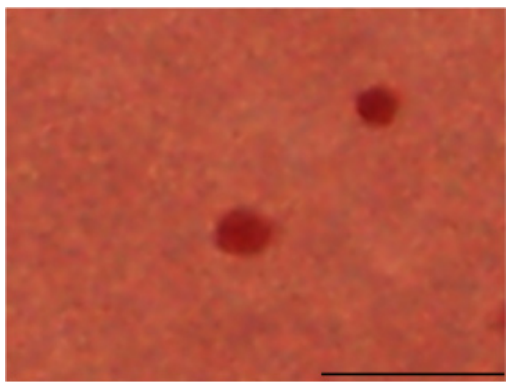

SF/AgS 1.0

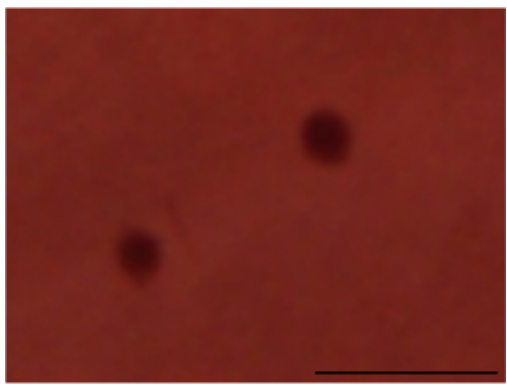

$100 \mu \mathrm{m}$
B

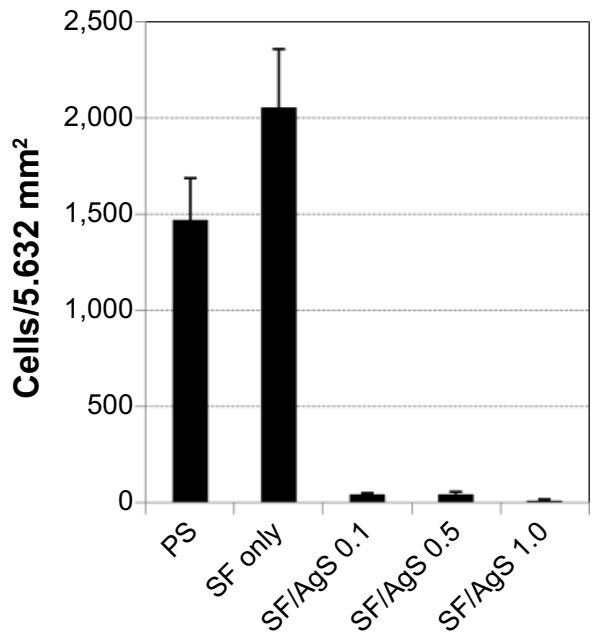

C

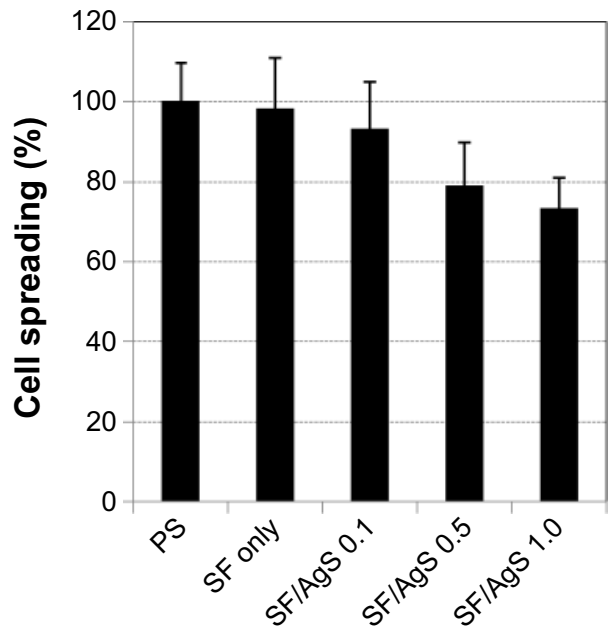

Figure 3 Effect of SF nanofibers containing SSD on attachment and spreading of NHEF.

Notes: (A) Representative images of NHEF attached to SF nanofibers containing SSD. (B) Numbers of NHEF adhering to SF nanofibers containing SSD. (C) Percentage of cell spreading of NHEF plated onto SF nanofibers containing SSD. Data are presented as the mean \pm standard deviation ( $n=4$ ).

Abbreviations: Ag, silver; NHEF, normal human epidermal fibroblasts; PS, polystyrene tissue culture plate; SSD, silver sulfadiazine; SF, silk fibroin; AgS 0.I, 0.I wt\% SSD; AgS 0.5, 0.5 wt\% SSD; AgS I.0, 1.0 wt\% SSD. 
and proliferation of keratinocytes in the epidermal layer of skin from the wound edge, but also by the differentiation of stem cells residing in the bulge of the hair follicles. ${ }^{23}$ Rapid re-epithelialization following skin wounding provides an optimum environment for wound healing, including a scaffold of cells and various growth factors that are indispensable for the wound healing process. Along with re-epithelialization, wound contraction plays a critical role in the early phase of wound healing by minimizing the open area through pulling the neighboring tissue toward the wound center. Alpha-smooth muscle actin generated from myofibroblasts plays a vital role in this process. Myofibroblasts arise by differentiation from fibroblasts and generate the force by which the wound area contracts during wound healing. ${ }^{24,25}$ Thus, intense research efforts are currently directed toward the development of new drugs and technologies to promote re-epithelialization and wound contraction. ${ }^{26}$

Acticoat, a wound dressing that employs nanocrystalline Ag technology, was reported to produce favorable results in an audit involving 70 burns patients who had few instances of cellulitis or antibiotic use. ${ }^{27}$ Other noncomparative studies have shown the safety and clinical benefits, such as less pain in burns patients using Acticoat wound dressings. ${ }^{28-30}$ When compared with topical antibiotic treatment in 20 patients who had meshed skin grafts, nanocrystalline Ag dressings have been shown to have an effect on epithelialization of their grafts. ${ }^{31}$

In the present study, wound healing effect between SF nanofibers containing SSD and Acticoat was compared using an SD rat model. Thirty-six SD rats were randomly divided into six groups of six rats each, which were subjected to the following six treatments: SF nanofibers only (no SSD; SF only), SF/AgS 0.1, SF/AgS 0.5, SF/AgS 1.0, Acticoat, or no treatment (control). The SD rat models treated by the surgical wounding protocol were photographed at days 3, 7, and 14 post wounding. The wound appearance in rats at day 3 post treatment is shown in Figure 4.

The earliest histological change in the wound bed after wounding is wound contraction, a vital process for
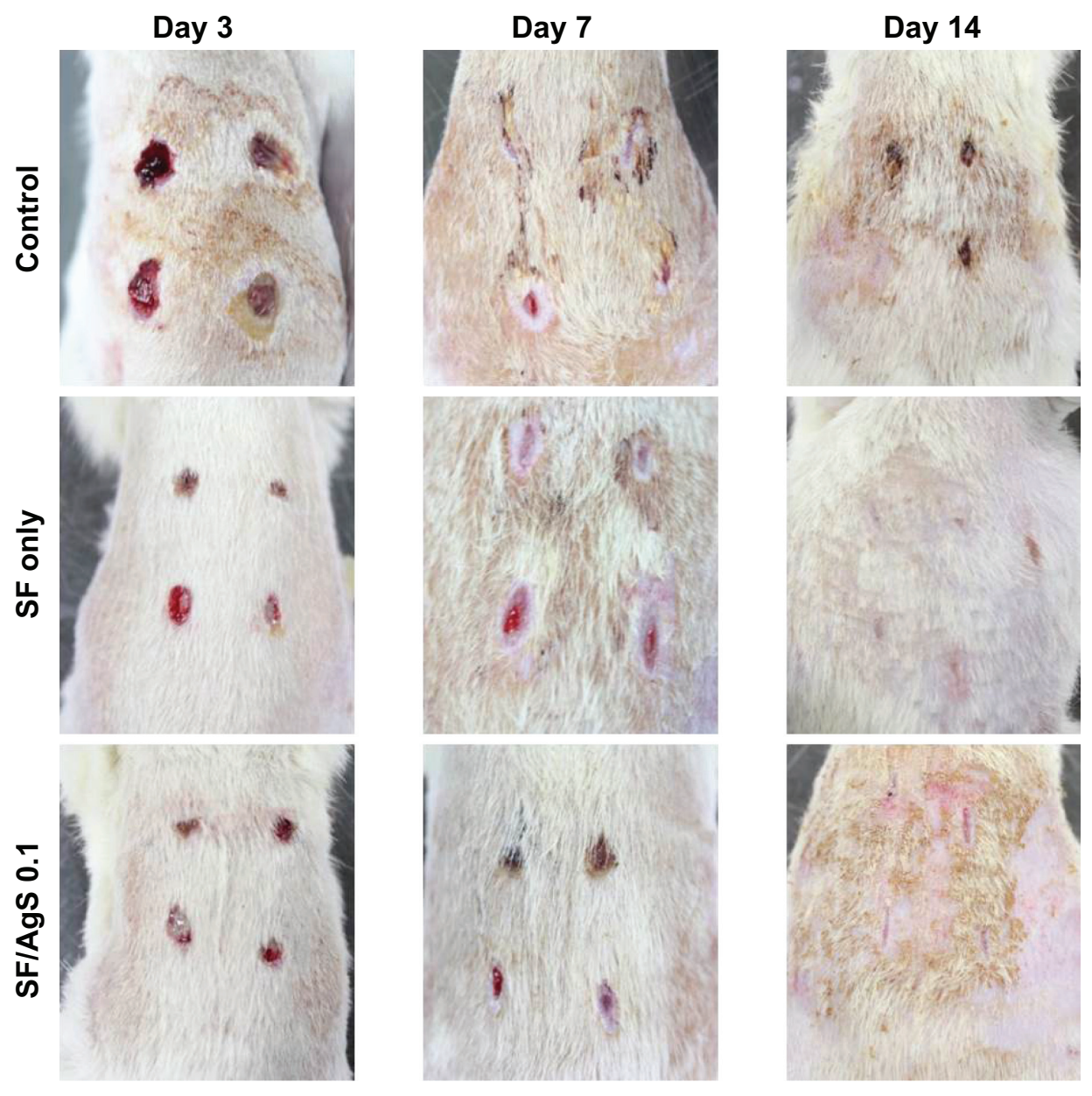

Figure 4 (Continued) 

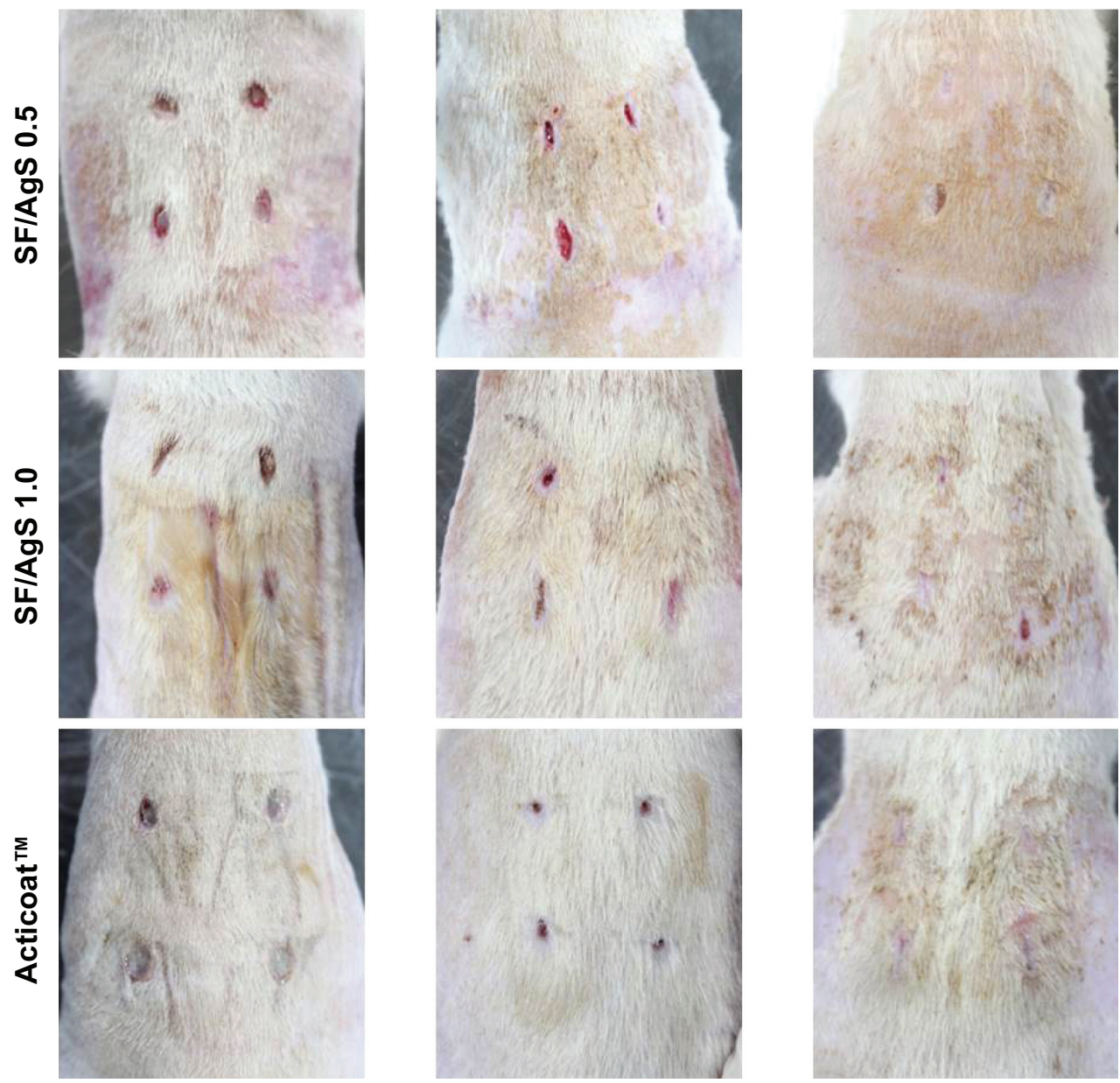

Figure 4 Wound appearances treated with Ag-based wound dressings at days 3, 7, and I 4 post wounding. Abbreviations: Ag, silver; SSD, silver sulfadiazine; SF, silk fibroin; AgS 0.I, 0.1 wt\% SSD; AgS 0.5, 0.5 wt\% SSD; AgS I.0, I.0 wt\% SSD.

minimizing the open wound area. Wound contraction is initiated by activated fibroblasts located at the wound edge, which differentiate into myofibroblasts that secrete actin and elastic fibers for wound contraction. ${ }^{26}$

At day 3 post surgery, limited wound healing without much wound contraction was observed in the controls (no Ag-based dressings). In contrast, those with Ag-based dressings presented wound healing, with scabs generated in the wounds treated with SF/AgS 1.0 and Acticoat. At day 7 post surgery, the wound healing behavior differed significantly from that observed at day 3. All groups showed wound closing and regenerated epidermis. In addition, the sizes of wounds in rats treated with $\mathrm{SF} / \mathrm{AgS} 1.0$ and Acticoat were much smaller than those in rats from the other treatment groups. At day 14 post surgery, complete wound contraction occurred and the wounds were filled with regenerating skin similar to the original skin in all groups except the control group. Results for the wound closure rate according to time after wounding are summarized in Figure 5. The rate of wound closure in rats treated with SF nanofibers containing SSD was significantly $(P<0.05)$ higher than that in the control group. However, the wound healing ability of SF/AgS 1.0 was similar to that of Acticoat containing nanocrystalline Ag.

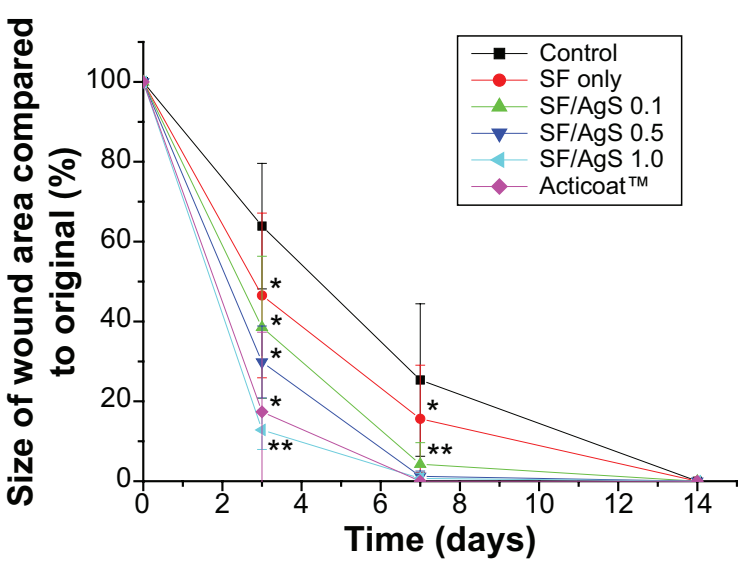

Figure 5 Rate of wound closure with time after wounding.

Notes: Data are presented as the mean \pm standard deviation $(n=4)$. $* P<0.05 ; * * P<0.00$ I. Abbreviations: $\mathrm{Ag}$, silver; SSD, silver sulfadiazine; SF, silk fibroin; AgS 0.1, 0.1 wt\% SSD; AgS 0.5, 0.5 wt\% SSD; AgS I.0, I.0 wt\% SSD. 


\section{Histological examination}

The results for histological examination of epithelium taken from the wounds at days 3, 7, and 14 post wounding are summarized in Figure 6. Hematoxylin and eosin staining showed that the epithelium at the edges of all wounds migrated toward the center. The histological morphology of wound healing was clearly observed at the wound margin by 3 days post wounding (Figure 6). At 3 days post wounding, the epidermis was regenerated in the wounds of the groups treated with $\mathrm{SF} / \mathrm{AgS} 1.0$ and Acticoat. At 7 days post wounding, regenerated epidermis was observed in all groups, and their dermal layers were thicker when compared with those at day 3 post wounding. Wounds treated with $\mathrm{SF} / \mathrm{AgS} 1.0$ or Acticoat showed faster healing behavior than the other treatment groups. At 14 days post wounding, the dermis and epidermis of the wounds was regenerated similar to that of the normal skin surrounding the wound. The extent of re-epithelialization in the wounds healed at 7 days post wounding for all six treatment groups is summarized in Figure 7. The extent of re-epithelialization of wounds treated with $\mathrm{SF} / \mathrm{AgS} 0.5$ or SF/AgS 1.0 was significantly $(P<0.05)$ higher than in those treated with Acticoat.

\section{Conclusion}

SF nanofibers containing SSD were prepared by electrospinning. The number of NHEK and NHEF attached to the SF nanofibers containing SSD decreased when the concentration of SSD increased, with the number of attached NHEF being lower than that of NHEK. In the animal study, the rate of wound closing was faster in rat treated with SSD containing a higher concentration of Ag. The wound healing effect of the SF nanofibers containing $1.0 \mathrm{wt} \%$ SSD was found to be comparable with that of Acticoat, a commercially available wound dressing. Therefore, the cytotoxic effects of SF nanofibers with SSD should be also considered in the development of Ag-release dressings for wound healing. It is a challenge to design wound dressings that maximize antimicrobial activity and minimize cellular toxicity.

\section{Day 3}
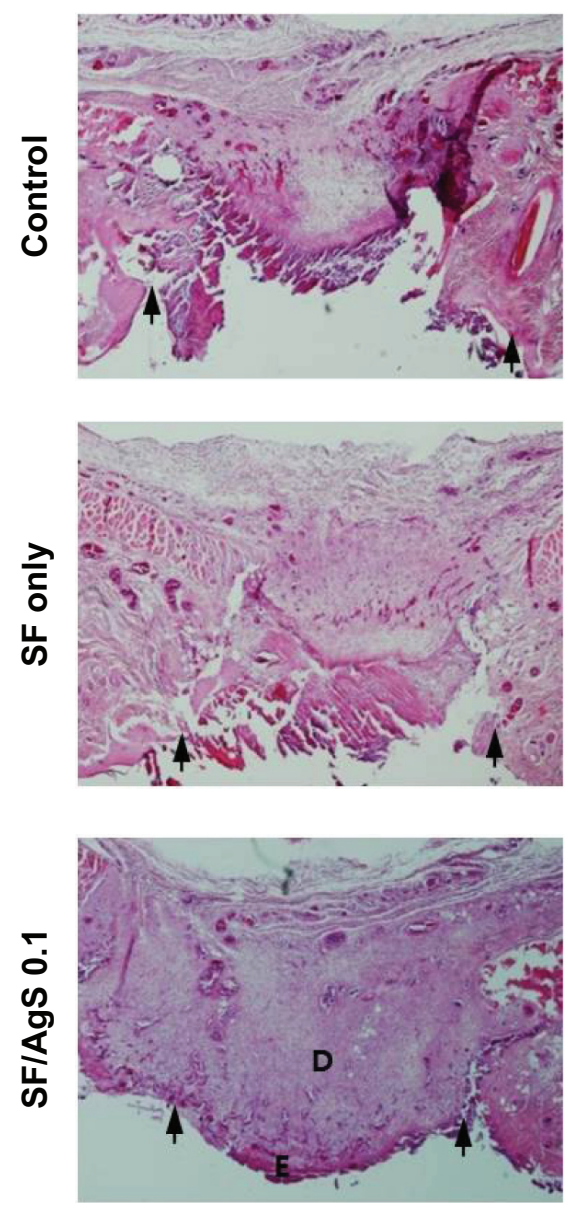

Day 7
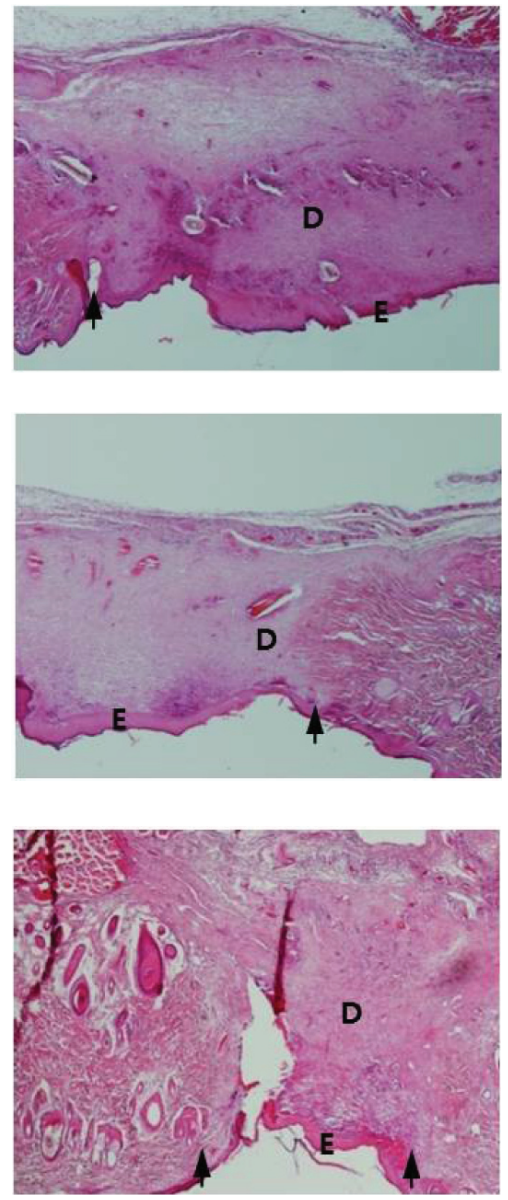

Day 14
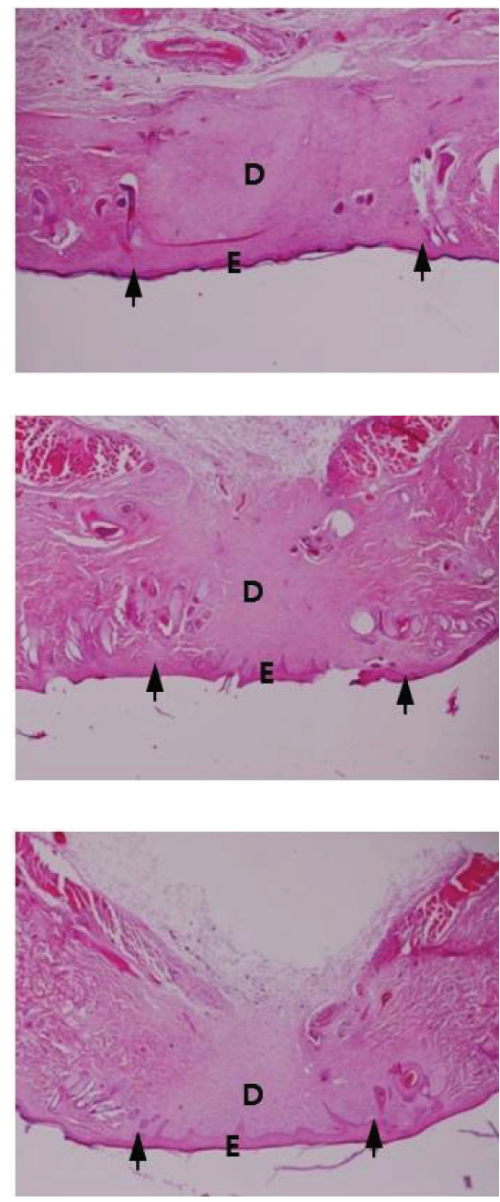

Figure 6 (Continued) 

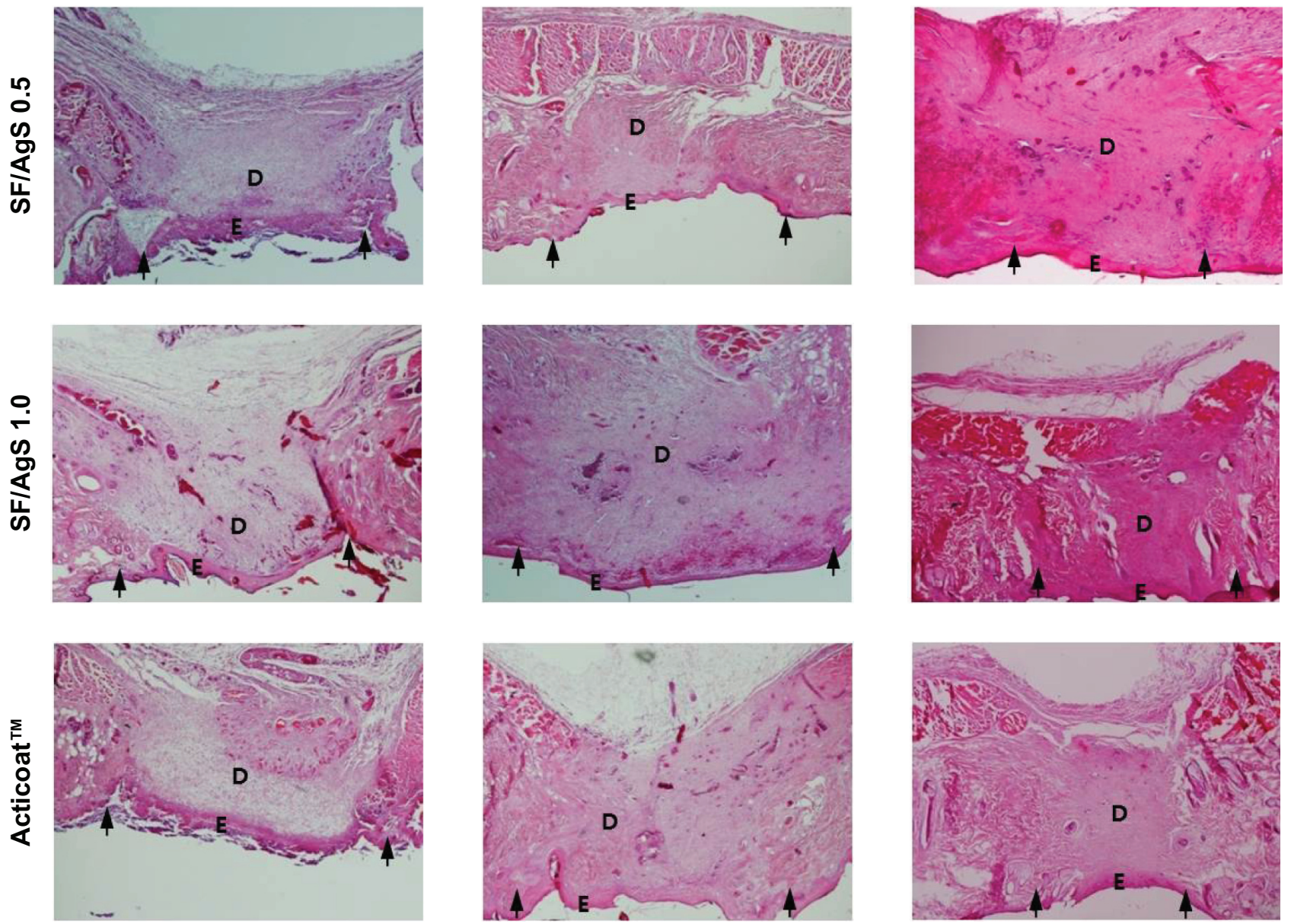

Figure 6 Histological morphology of healed wounds in rats at days 3, 7, and I4 post wounding.

Notes: D, regenerated dermis; E, regenerated epidermis; arrows indicate wound edge.

Abbreviations: Ag, silver; SSD, silver sulfadiazine; SF, silk fibroin; AgS 0.I, 0.1 wt\% SSD; AgS 0.5, 0.5 wt\% SSD; AgS I.0, I.0 wt\% SSD.

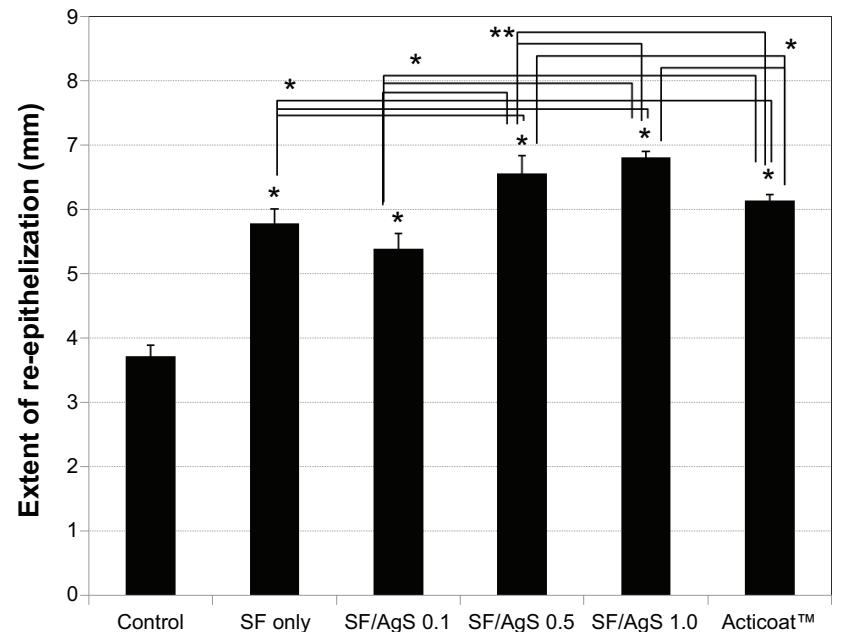

Figure 7 Extent of re-epithelialization measured histologically on wounds healed for 7 days.

Notes: Data are presented as the mean \pm standard deviation $(n=4) * P<0.001 ; * * P<0.05$. Abbreviations: Ag, silver; SSD, silver sulfadiazine; SF, silk fibroin; AgS 0.I, 0.I wt\% SSD; AgS 0.5, 0.5 wt\% SSD; AgS I.0, 1.0 wt\% SSD.

\section{Acknowledgment}

This research was supported by the Nuclear $\mathrm{R}$ and $\mathrm{D}$ program (NRF-2012M2A2A6035747) through the National Research Foundation funded by the Ministry of Science, ICT and Future Planning, Korea.

\section{Disclosure}

The authors report no conflicts of interest in this work.

\section{References}

1. Klasen HJ. A historical review of the use of silver in the treatment of burns. I. Early uses. Burns. 2000;26(2):117-130.

2. Moyer CA, Brentano L, Gravens DL, Margraf HW, Monafo WW. Treatment of large human burns with 0.5 percent silver nitrate solution. Arch Surg. 1965;90(6):812-867.

3. Klasen HJ. A historical review of the use of silver in the treatment of burns. II. Renewed interest for silver. Burns. 2000;26(2):131-138.

4. Bellinger CG, Conway H. Effect of silver nitrate and sulfamylon on epithelial regeneration. Plast Reconstr Surg. 1970;45:582-585. 
5. Rai M, Yadav A, Gade A. Silver nanoparticles as a new generation of antimicrobials. Biotechnol Adv. 2009;27(1):76-83.

6. Stanford W, Rappole BW, Fox CL. Clinical experience with silver sulfadiazine, a new topical agent for control of Pseudomonas infections in burns. $J$ Trauma. 1969;9(5):377-388.

7. Fox CL, Modak SM. Mechanism of silver sulfadiazine action on burn wound infection. Antimicrob Agents Chemother. 1974;5(6):582-588.

8. Burd A, Kwok CH, Hung SC, et al. A comparative study of the cytotoxicity of silver-based dressings in monolayer cell, tissue explant, and animal model. Wound Repair Regen. 2007;15(1):94-104.

9. Lok CN, Ho CM, Chen R, et al. Proteomic analysis of the mode of antibacterial action of silver nanoparticles. J Proteome Res. 2006;5(4):916-924.

10. Lansdown $\mathrm{AB}$. A guide to the properties and uses of silver dressings in wound care. Prof Nurse. 2005;20(5):41-43.

11. Dunn K, Edwards-Jones V. The role of Acticoat with nanocrystalline silver in the management of burns. Burns. 2004;30 Suppl 1:S1-S9.

12. Yu DG, Zhou J, Chatterton NP, et al. Polyacrylonitrile nanofibers coated with silver nanoparticles using a modified coaxial electrospinning process. Int J Nanomedicine. 2012;7:5725-5732.

13. Draelos ZD. The cosmeceutical realm. Clin Dermatol. 2008;26(6): 627-632.

14. Vepari C, Kaplan DL. Silk as a biomaterial. Prog Polym Sci. 2007; 32(8-9):991-1007.

15. Kasoju N, Bora U. Silk fibroin in tissue engineering. Adv Healthc Mater. 2012;1(4):393-412.

16. Yan S, Zhang Q, Wang J, et al. Silk fibroin/chondroitin sulfate/ hyaluronic acid ternary scaffolds for dermal tissue reconstruction. Acta Biomater. 2013;9(3):6771-6782.

17. Kundu B, Rajkhowa R, Kundu SC, et al. Silk fibroin biomaterials for tissue regenerations. Adv Drug Deliv Rev. 2013;65(4):457-470.

18. Min BM, Lee G, Kim SH, et al. Electrospinning of silk fibroin nano fibers and its effects on the adhesion and spreading of normal human keratinocytes and fibroblasts in vitro. Biomaterials. 2004;25(7-8):1289-1297.

19. Jeong L, Yeo I, Kim HN, et al. Plasma-treated silk fibroin nanofibers for skin regeneration. Int J Biol Macromol. 2009;44(3):222-228.
20. Mould AP, Askari JA, Humphries MJ. Molecular basis of ligand recognition by integrin alpha 5 beta 1 . I. Specificity of ligand binding is determined by amino acid sequences in the second and third $\mathrm{NH}_{2}$-terminal repeats of the alpha subunit. $J$ Biol Chem. 2000;275(27):20324-20336.

21. Chun JY, Kang HK, Jeong L, et al. Epidermal cellular response to poly(vinyl alcohol) nanofibers containing silver nanoparticles. Colloids Surf B Biointerfaces. 2010;78(2):334-342.

22. Martin P. Wound healing-aiming for perfect skin regeneration. Science. 1997;276(5309):75-81.

23. Nagato H, Umebayashi Y, Wako M, Tabata Y, Manabe M. Collagenpoly glycolic acid hybrid matrix with basic fibroblast growth factor accelerates angiogenesis and granulation tissue formation in diabetic mice. J Dermatol. 2006;33(10):670-675.

24. Jester JV, Petroll WM, Cavanagh HD. Corneal stromal wound healing in refractive surgery: the role of myofibroblasts. Prog Retin Eye Res. 1999;18(3):311-356.

25. Feugate JE, Li Q, Wong L, Martin-Green M. The cxc chemokine cCAF stimulates differentiation of fibroblasts into myofibroblasts and accelerates wound closure. J Cell Biol. 2002;156(1):161-172.

26. Liu X, Lee PY, Ho CM, et al. Silver nanoparticles mediate differential reponses in keratinocytes and fibroblasts during skin wound healing. ChemMedChem. 2010;5(3):468-475.

27. Rustogi R, Milm J, Frazer JF, Kimble RM. The use of Acticoat in neonatal burns. Burns. 2005;31(7):878-882.

28. Demling RH, DeSanti MDL. The role of silver technology in wound healing: Part 1: Effects of silver on wound management. Wounds. 2001;13 Suppl A:4-15.

29. Kunimoto BT. Management and prevention of venous leg ulcers: a literature-guided approach. Ostomy Wound Manag. 2001;47(5): $38-53$.

30. Demling RH, DeSanti MDL. The rate of re-epithelialization across meshed skin grafts is increased with exposure to silver. Burns. 2002;28(3):264-266.

31. Moiemen NS, Shale E, Drysdale KJ, et al. Acticoat dressings and major burns: systemic silver absorption. Burns. 2011;37(1):27-35.
International Journal of Nanomedicine

\section{Publish your work in this journal}

The International Journal of Nanomedicine is an international, peerreviewed journal focusing on the application of nanotechnology in diagnostics, therapeutics, and drug delivery systems throughou the biomedical field. This journal is indexed on PubMed Central, MedLine, CAS, SciSearch ${ }^{\circledR}$, Current Contents ${ }^{\circledR} /$ Clinical Medicine,

\section{Dovepress}

Journal Citation Reports/Science Edition, EMBase, Scopus and the Elsevier Bibliographic databases. The manuscript management system is completely online and includes a very quick and fair peer-review system, which is all easy to use. Visit http://www.dovepress.com/ testimonials.php to read real quotes from published authors. 